\title{
РОЛЬ ОФЭКТ/КТ В ДИАГНОСТИКЕ И СТАДИРОВАНИИ ДИФФЕРЕНЦИРОВАННОГО РАКА ЩИТОВИДНОЙ ЖЕЛЕЗЫ
}

\author{
Хайдарова Ф.А., Нурмухамедов Д.Б.
}

Республиканский специализированный научно-практический медицинский центр эндокринологии, Ташкент, узбекистан

ОФЭКТ/КТ признана в настоящее время как эффективная модальность диагностики и мониторинга лечения дифференцированного рака щитовидной железы (ДРЩЖ). Диагностическая точность ОФЭКТ/КТ в стадирование ДРщЖ оценивается неоднозначно.

ЦЕЛЬ: оценка диагностической ценности ОФЭКТ/КТ в выявлении и стадирование дифференцированного рака щитовидной железы в сравнении с данными ультрасонографии.

МАТЕРИАЛЫ И МЕТОДЫ: обследовано 50 больных с клиническими признаками узловых образований в щитовидной железе, из них 35 женщины, 15 мужчины. В качестве специальных методов исследования щитовидной железы использованы ОФЭКТ/КТ, ультрасонография и тонкоигольная аспирационная биопсия с цитологическим исследованием. 36 больных подвергнуты хирургическому лечению с морфологическим исследованием удаленных тканей. ОФЭКТ/КT выполнялась на SPECT/CT Mediso AnyScan (Венгрия), спустя 24 часа после введения радиоактивного индикатора.

PЕЗУЛЬТАТЫ: согласно данным патогистологического анализа, ДРЩЖ был обнаружен у 36 из 50 обследованных больных. С учетом морфологически верифицированного диагноза показатели диагностической эффективности ОФЭКТ /КТ составили: чувствительность 88,9\%, специфичность 70\%, диагностическая точность 83\%. Локально-региональная стадия распространенности опухоли была правильно определена при ОФЭКТ/ КТ у 83,3\% больных ДРщЖ. Эффективность ультрасонографии в выявлении и дифференциации рака и доброкачественных образований щитовидной железы почти не отличалась от ОФЭКТ/КТ (чувствительность 90\%, специфичность 77\% и диагностическая точность 86\%), но ОФЭКТ/КТ была более точной в обнаружении поражения шейных лимфоузлов и дистанционных метастазов, и, таким образом, в стадирование ДРщЖ.

ВЫВОДЫ: ОФЭКТ/КТ является ценной модальностью визуализации для диагностики и стадирования ДРЩЖ. Возможность совмещения анатомической и функционально-метаболической визуализации является особым преимуществом ОФЭКТ/КТ в качестве средства мониторинга лечения ДРЩЖ. 